\title{
Survival of a Patient with Amniotic Fluid Embolism Based on Multidisciplinary Team Training: A Case Report of a Swiss University Hospital
}

\author{
F. Theintz ${ }^{1}$, G. Haller ${ }^{2}$, R. E. Pfister ${ }^{1}$, B. Martinez de Tejada ${ }^{1}$, I. Eperon ${ }^{1}$ \\ ${ }^{1}$ Department of Women-Children-Teenagers, University Hospitals of Geneva, Geneva, Switzerland \\ ${ }^{2}$ Department of Anesthesia, University Hospitals of Geneva, Geneva, Switzerland \\ Email: ^begona.martinezdetejada@hcuge.ch
}

How to cite this paper: Theintz, F., Haller, G., Pfister, R.E., de Tejada, B.M. and Eperon, I. (2019) Survival of a Patient with Amniotic Fluid Embolism Based on Multidisciplinary Team Training: A Case Report of a Swiss University Hospital. Open Journal of Obstetrics and Gynecology, 9, 216-222.

https://doi.org/10.4236/ojog.2019.92022

Received: January 18, 2019

Accepted: February 16, 2019

Published: February 19, 2019

Copyright $\odot 2019$ by author(s) and Scientific Research Publishing Inc. This work is licensed under the Creative Commons Attribution International License (CC BY 4.0).

http://creativecommons.org/licenses/by/4.0/

\begin{abstract}
Amniotic fluid embolism (AFE) is a rare, unpredictable and unpreventable event with high maternal and fetal morbidity and mortality. Its clinical presentation is nonspecific ranging from moderate organ dysfunction to cardiovascular collapse with disseminated intravascular coagulation (DIC), which can lead to death. In a situation of severe systemic disease, maternal survival is largely dependent on the combined efficacy of gynecological and anesthetic teams. We report a case of AFE in a healthy woman admitted to the delivery room for labor induction. Due to a loss of consciousness soon after epidural anesthesia associated to fetal bradycardia, an emergency caesarean section was performed which was complicated by uterine atony and DIC requiring hysterectomy. The outcome was favorable due to fast and efficient multidisciplinary care, emphasizing the benefit of having been trained for such situations through simulation and team training programs for obstetrical emergency management.
\end{abstract}

\section{Keywords}

Amniotic Fluid Embolism, Obstetrics, Simulation, Team Training

\section{Introduction}

Amniotic fluid embolism (AFE) is a rare, unpredictable and unpreventable event with high maternal and fetal morbidity and mortality. According to literature, its incidence is difficult to establish due to the various criteria used to assess the diagnosis [1] [2]. Moreover, AFE clinical presentation can range from moderate 
organ dysfunction to cardiovascular collapse with disseminated intravascular coagulation (DIC), which can be mistaken with other conditions [2] [3]. In a situation of severe systemic disease, maternal survival is largely dependent on the combined efficacy of gynecological and anesthetic teams.

We report a case of AFE with favorable outcome due to fast and efficient multidisciplinary care, emphasizing the benefit of having been trained for such emergency situations. Over the last few years, publications describing simulation-based training programs for obstetric crisis have proved to be efficient and allow better recognition and care of critical obstetric situations [4].

\section{Case Presentation}

A 31-year-old healthy primigravida at 40 weeks of gestation was admitted to the delivery room for labor induction due to fetal macrosomia. She had cervical ripening with a Foley catheter for 24 hours followed by oxytocin administration. She had artificial rupture of the membranes. Five hours later, she had spinal-epidural analgesia to relieve pain due to uterine contractions. Cervical dilation was $2 \mathrm{~cm}$. Soon after the initial bolus administration of $1 \mathrm{~mL}$ of $0.25 \%$ bupivacaine and $20 \mu \mathrm{g}$ fentanyl in the intrathecal space, the patient complained of nausea. Hypotension $(90 / 39 \mathrm{mmHg})$, tachycardia (108/minute) and cyanosis were also noticed. The fetus was bradycardic $(60 \mathrm{bpm})$ despite resuscitation measures that included phenylephrine administration $(300 \mu \mathrm{g})$, oxytocin discontinuation, left lateral decubitus position and oxygen supply. Soon after, the patient lost consciousness and required an emergency caesarean section for fetal-maternal rescue.

The baby was extracted 5 minutes later and was hypotonic and unresponsive. He weighed 4250 grams. Apgar score was 0, 4, and 4 at 1, 5 and 10 minutes, respectively. Arterial $\mathrm{pH}$ was 6.69 , and venous $\mathrm{pH}$ was 6.73 . Immediate neonatal resuscitation, including bag and mask ventilation for 20 minutes, was required followed by continuous nasal positive airway pressure (CPAP) for additional 20 minutes.

The uterus remained atonic and unresponsive to massage and intravenous administration of uterotonics [oxytocin in first line ( 25 minutes) followed by sulprostone and methylergometrine]. Blood loss was estimated at 3 liters, and clinically abnormal coagulation was noted. Forty minutes after initiation of surgery, blood tests showed anemia (99 g/L), thrombocytopenia $(63 \mathrm{G} / \mathrm{L})$ and severely disturbed coagulation (Quick $<5 \%$, INR $>11$, PTT $>160$ s, fibrinogen $<$ $0.5 \mathrm{~g} / \mathrm{L}$ ), indicating the presence of DIC (Table 1). Clinical and biological features pointed to the diagnosis of amniotic fluid embolism, which triggered a specific emergency routine requiring immediate and aggressive intervention via efficient collaborative work between obstetric and anesthetic teams.

As a result, aggressive blood and coagulation factors transfusion was initiated. Given that hemorrhage could not be controlled ( $3 \mathrm{~L}$ blood loss), an emergency hysterectomy was performed. The patient received 13 liters of crystalloids, 17 units of packed red blood cells, 12 units of fresh frozen plasma, 3 platelet units, 
Table 1. Blood test evolution during management of our patient.

\begin{tabular}{ccc}
\hline & During operation & 1 hour after operation \\
\hline Hemoglobin (g/L) & 99 & 82 \\
Platelet (G/L) & 63 & 57 \\
Quick (\%) & $<5$ & 74 \\
INR & $>11$ & 1.14 \\
PTT (sec) & $>160$ & 42.8 \\
Fibrinogen (g/L) & $<0.5$ & 0.8 \\
\hline
\end{tabular}

1200 UI of combined coagulation factors (Prothromplex), $11 \mathrm{mg}$ of recombinant factor VIIa, $10 \mathrm{~g}$ of tranexamic acid and $12 \mathrm{~g}$ of fibrinogen in total. At the end of surgery, bleeding was controlled, and blood pressure was maintained with noradrenalin at a mean of $70 \mathrm{mmHg}$. She was transferred to the intensive care unit. Eight hours after the first intervention, she required a second-look surgery due to massive blood exteriorization in drains. Blood clots were removed, but no active bleeding was identified. Close follow-up was performed during her hospital stay: the outcome was favorable allowing mother and baby to return home after 11 days. A control was organized with six weeks after surgery by the attending physician. At follow-up six months later, both mother and child were healthy.

\section{Discussion}

In the USA, amniotic fluid embolism (AFE) incidence is estimated as 2 $8 / 100,000$ deliveries and is responsible for $7.5 \%-10 \%$ of maternal deaths [5]. In Australia-New Zealand, AFE has an estimated incidence of 5.4/100,000 deliveries with a mortality rate of $0.8 / 100,000$ [6].

The diagnosis of AFE is difficult as clinical signs are often nonspecific ranging from mild organ dysfunction to sudden cardiac arrest [1]. Clark et al. suggest the use of standardized criteria to diagnose AFE [2]. These criteria include the following:

1) Sudden cardio-respiratory arrest or severe hypotension (systolic BP $<90$ $\mathrm{mmHg}$ ) associated with breathing difficulties (dyspnea, cyanosis, $\mathrm{O}_{2}$ saturation $<$ 90\%).

2) Documented disseminated intravascular coagulation (DIC) following the manifestation of the first symptoms or signs using the ISTH (International Society on Thrombosis and Haemostasis) scoring system modified for pregnancy as shown in Table 2 [2].

3) Onset of symptoms during labor or within 30 minutes following placental delivery.

4) Absence of fever during labor.

AFE is caused by the entry of amniotic fluid into maternal circulation following a rupture of the utero-placental barrier. The resulting mechanical obstruction and pulmonary vasoconstriction are responsible for the initial hypoxemia 
Table 2. Modified international society on thrombosis and Haemostasis scoring system for overt disseminated intravascular coagulation in pregnancy [2].

- Platelet count: $>100,000 / \mathrm{mL}=0,<100,000 / \mathrm{mL}=1,<50,000 / \mathrm{mL}=2$

- Prolonged prothrombin time or international normalized ratio: $<25 \%$ increase $=0,25 \%-50 \%$ increase $=1,>50 \%$ increase $=2$

- Fibrinogen level: $>200 \mathrm{mg} / \mathrm{L}=0,<200 \mathrm{mg} / \mathrm{L}=1$

A score $\geq 3$ is compatible with overt disseminated intravascular coagulation in pregnancy

and hypotension. Left ventricular failure seen in severe cases can lead to pulmonary edema and occasionally cardiac arrest. The presence of amniotic cells in maternal circulation triggers an acute inflammatory cascade, resulting in severe coagulation disorders. However, the presence of amniotic fluid in the maternal circulation does not always result in severe organ dysfunction and systemic inflammation, as shown by Kuhlman et al. and Clark et al. [7] [8]. This finding suggests immuno-mediated mechanisms that can trigger the inflammatory cascade depending on individual susceptibility. When inflammation occurs, a number of biomarkers, such as complement fractions C3-C4 and serum tryptase, can be identified in maternal blood [9]. These markers are not specific as elevated tryptase in anaphylactic reactions [1]. To date, IGF-BP1 (insulin-like growth factor binding protein-1) appears to be the only marker specific for AFE [10]. Other markers include amniotic fluid cells identified in maternal blood. In our patient, the search for amniotic cells in the maternal blood was negative, and the vales of IGF-BP1 and serum tryptase were in the normal range. The diagnosis of AFE remains therefore based on suggestive clinical features and severe disseminated intravascular coagulation (meeting all 4 criteria proposed by Clark). This case demonstrates that suspicion of AFE should be managed as a major emergency situation within a multidisciplinary labor ward team providing obstetrical, anesthetic intensive care and neonatology expertise.

Despite the gravity of symptoms, maternal mortality due to AFE has decreased by more than $50 \%$ since the 80 s with a mortality rate ranging from 11 to $48 \%$ according to various authors [1]. This has been made possible by the development of resuscitation techniques and team training in the management of emergency obstetrical situations as shown in our report. Simulation-based training programs in University Hospitals of Geneva include a case of cardiovascular collapse requiring an emergency caesarean section that may be comparable with our clinical case. The multidisciplinary team is trained to work together to achieve the steps described below.

If AFE occurs during labor, resuscitation measures should essentially be taken to maintain the best possible utero-placental flow and fetal vitality (left lateral position, displacement of the uterus to the left). In situations of cardiovascular maternal collapse, the fetal cardiac rhythm is inevitably impaired, requiring an emergency caesarean section for fetal-maternal rescue followed by stabilization of vital functions via the following mechanisms: 1) Maintenance of the respiratory system varies from simple oxygen mask supply to orotracheal intubation 
using various mechanical ventilation procedures. 2) Hypotension and cardiac failure may require plasma volume expanders, vasopressors and inotropic drugs. In case of refractory cardiogenic shock, a veno-arterial ECMO (extracorporeal membrane oxygenation) can be performed if available within the hospital setting. By contrast, if the failure is predominantly respiratory with hypoxemia refractory to mechanical ventilation, ECMO should be veno-venous [11]. It should be noted that anticoagulant drugs required to implement ECMO can worsen blood losses in women suffering from coexisting blood dyscrasia. This therapeutic option should therefore be assessed on a case-by-case basis [12]. 3) Hemorrhagic blood losses are compensated by vascular filling and packed red blood cells. The associated uterine atony should be treated by uterotonics as the first line possibly combined with intrauterine balloon tamponade. In more severe cases, uterine artery embolization must be considered if the patient is hemodynamically stable. If not, the remaining options include uterine artery ligation or hysterectomy hemostasis. For all cases of postpartum hemorrhage, an institutional management protocol jointly developed by the Swiss Society of Gynecology and Obstetrics and the Swiss Society of Anesthesiology and Resuscitation is used [13]. This protocol is yearly rehearsed by all staff members of the labor unit (midwives, obstetricians, nurses and anesthetists) during simulation sessions that reproduce different scenarios of problematical deliveries and emergencies. All staff is also trained in human factor management through a mandatory classroom team-training program. 4) Blood dyscrasia is treated with the administration of tranexamic acid, fibrinogen, thrombapheresis, fresh frozen plasma and coagulation factors. Recombinant factor VIIa is used as a last resort when hemorrhage cannot be controlled by other means as this factor favors the development of diffuse thrombosis and secondary organ damage. We would like to emphasize the importance to prolong treatment to correct hemodynamic status and coagulation defects for longer than usual after surgery since mechanism of coagulation dysfunction persists for many hours. In our case, aggressive early management after the first surgery may avoid a second surgery.

Maternal and fetal outcomes depend on condition severity and rapidity of care. Neonatal mortality rate may reach $40 \%$ and the major neonatal complication is hypoxemic-ischemic encephalopathy with impaired long-term neurological outcomes [12]. Today, early therapeutic hypothermia may improve the neurologic prognosis. The key to the best maternal and fetal outcome in critical situations depends on fast multidisciplinary management [3]. Simulation-based team training appears particularly beneficial for emergency obstetrical care and appears of critical importance for complex multidisciplinary approaches.

\section{Conclusion}

AFE is an unpreventable, dramatic and rare obstetrical event. The reactivity and competence of a trained multidisciplinary team will determine the outcomes of the mother and baby. A high degree of suspicion should exist when hypotension, hypoxia and coagulopathy are present and should prompt rapid reaction of a 
multidisciplinary team. Reduction in mortality can be achieved thanks to medical progress but especially due to simulation programs, allowing for rapid diagnosis and management.

\section{Conflicts of Interest}

The authors declare no conflicts of interest regarding the publication of this paper.

\section{References}

[1] McDonnell, N.J., Percival, V. and Paech, M.J. (2013) Amniotic Fluid Embolism: A Leading Cause of Maternal Death Yet Still a Medical Conundrum. International Journal of Obstetric Anesthesia, 22, 329-336. https://doi.org/10.1016/j.ijoa.2013.08.004

[2] Clark, S.L., Romero, R., Dildy, G.A., Callaghan, W.M., Smiley, R.M., Bracey, A.W., et al. (2016) Proposed Diagnostic Criteria for the Case Definition of Amniotic Fluid Embolism in Research Studies. American Journal of Obstetrics and Gynecology, 215, 408-412. https://doi.org/10.1016/j.ajog.2016.06.037

[3] Society for Maternal-Fetal Medicine (SMFM), Pacheco, L.D., Saade, G., Hankins, G.D. and Clark, S.L. (2016) Amniotic Fluid Embolism: Diagnosis and Management. American Journal of Obstetrics and Gynecology, 215, B16-B24.

[4] Robertson, B., Schumacher, L., Gosman, G., Kanfer, R., Kelley, M. and DeVita, M. (2009) Simulation-Based Crisis Team Training for Multidisciplinary Obstetric Providers. Simulation in Healthcare: The Journal of the Society for Simulation in Healthcare, 4, 77-83. https://doi.org/10.1097/SIH.0b013e31819171cd

[5] Fong, A., Chau, C.T., Pan, D. and Ogunyemi, D.A. (2015) Amniotic Fluid Embolism: Antepartum, Intrapartum and Demographic Factors. The Journal of Maternal-Fetal \& Neonatal Medicine, 28, 793-798. https://doi.org/10.3109/14767058.2014.932766

[6] McDonnell, N., Knight, M., Peek, M.J., Ellwood, D., Homer, C.S., McLintock, C., et al. (2015) Amniotic Fluid Embolism: An Australian-New Zealand Population-Based study. BMC Pregnancy Childbirth, 15, 352.

https://doi.org/10.1186/s12884-015-0792-9

[7] Kuhlman, K., Hidvegi, D., Tamura, R.K. and Depp, R. (1985) Is Amniotic Fluid Material in the Central Circulation of Peripartum Patients Pathologic? American Journal of Perinatology, 2, 295-299. https://doi.org/10.1055/s-2007-999974

[8] Clark, S.L., Pavlova, Z., Greenspoon, J., Horenstein, J. and Phelan, J.P. (1986) Squamous Cells in the Maternal Pulmonary Circulation. American Journal of Perinatology, 154, 104-106. https://doi.org/10.1016/0002-9378(86)90402-3

[9] Busardò, F.P., Frati, P., Zaami, S. and Fineschi, V. (2015) Amniotic Fluid Embolism Pathophysiology Suggests the New Diagnostic Armamentarium: $\beta$-Tryptase and Complement Fractions C3-C4 Are the Indispensable Working Tools. International Journal of Molecular Sciences, 16, 6557-6570. https://doi.org/10.3390/ijms16036557

[10] Legrand, M., Rossignol, M., Dreux, S., Luton, D., Ventré, C., Barranger, E., et al. (2012) Diagnostic Accuracy of Insulin-Like Growth Factor Binding Protein-1 for Amniotic Fluid Embolism. Critical Care Medicine, 40, 2059-2063. https://doi.org/10.1097/CCM.0b013e31824e6737

[11] Giraud, R. (2017) Amniotic Fluid Embolism and ECMO: Powerpoint Presentation. 
University Hospital of Geneva, Geneva.

[12] Knight, M., Berg, C., Brocklehurst, P., Kramer, M., Lewis, G., Oats, J., et al. (2012) Amniotic Fluid Embolism Incidence, Risk Factors and Outcomes: A Review and Recommendations. BMC Pregnancy Childbirth, 12, 7. https://doi.org/10.1186/1471-2393-12-7

[13] Bischofberger, A., Savoldelli, G.L. and Irion, O. (2011) Prise en charge multidisciplinaire des hémorragies du Post-Partum: Nouvelles strategies. Revue Médicale Suisse, 7, 334-339. 